Musées, Patrimoine et Culture scientifiques et techniques

$194 \mid 2021$

mars-avril 2021

\title{
Éclairer pour illuminer. L'éclairage, un dispositif muséal de médiation
}

\section{Viviana Gobbato et Daniel Schmitt}

\section{OpenEdition \\ Journals}

Édition électronique

URL : https://journals.openedition.org/ocim/4259

DOI : 10.4000/ocim.4259

ISSN : 2108-646X

Éditeur

OCIM

Édition imprimée

Date de publication : 1 mars 2021

Pagination : $32-37$

ISSN : 0994-1908

Référence électronique

Viviana Gobbato et Daniel Schmitt, «Éclairer pour illuminer. L'éclairage, un dispositif muséal de médiation », La Lettre de I'OCIM [En ligne], 194 | 2021, mis en ligne le 01 mars 2021, consulté le 11 avril 2022. URL : http://journals.openedition.org/ocim/4259 ; DOI : https://doi.org/10.4000/ocim.4259

Ce document a été généré automatiquement le 11 avril 2022.

Tous droits réservés 


\title{
Éclairer pour illuminer. L'éclairage, un dispositif muséal de médiation
}

\author{
Viviana Gobbato et Daniel Schmitt
}

Éclairage dynamique (installé en 2014) de la Grande Galerie de l'Évolution.

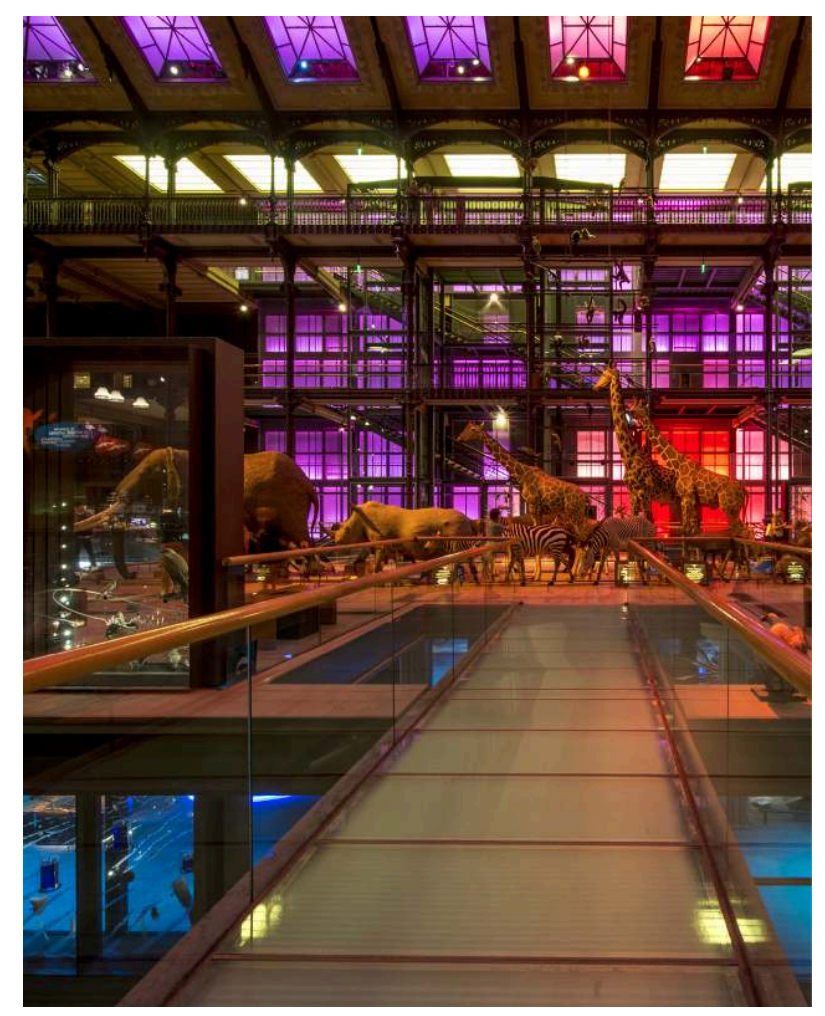

(c) MNHN/B. Jay

Le pilotage dynamique de l'éclairage offre aujourd'hui de nombreuses possibilités de modélisation et de contrôle de la lumière. Ces technologies peuvent guider le regard 
des visiteurs, leur faire vivre des nouvelles expériences esthétiques, sensibles et cognitives. L'éclairage dynamique peut participer d'une nouvelle forme de médiation.

\section{Lumière et musée, du danger à l'opportunité}

2 À l'exception de quelques expositions où le visiteur est volontairement plongé dans le noir, les musées ne peuvent se passer de lumière. A minima, les œuvres, le parcours de visite, les cartels et les textes nécessitent d'être éclairés. Convenir de l'importance de l'éclairage dans les musées relève d'un lieu commun, tant les muséographes s'en préoccupent depuis deux cents ans: la Grande Galerie du Louvre, le Musée de l'Orangerie et le Musée d'Orsay en sont quelques exemples parmi les plus connus. Ainsi, on pourrait même considérer que la question de l'éclairage et de sa mise en œuvre accompagne le musée depuis sa naissance. La lumière est déterminante quant à l'accessibilité dans l'exposition, le parcours dans les salles, la lisibilité des œuvres et des cartels. En un mot, la lumière est essentielle pour qu'il y ait « expérience de visite ».

La dimension potentiellement préjudiciable, voire néfaste de la lumière nourrit bien des échanges entre conservateurs, régisseurs et professionnels de l'éclairage. On demande aux projets d'éclairage de résoudre l'antagonisme entre présentation et conservation préventive des œuvres, en respectant des normes internationales (CIE, 2004). Ainsi, la lumière fascine tout autant qu'elle effraie: il faut la maitriser, la contrôler, la diriger. Cependant, depuis les années 2000, la technologie LED a permis de s'affranchir d'une partie des normes établies essentiellement pour les sources traditionnelles à incandescence et fluorescence. De plus, les protocoles d'éclairage dynamique - filaire et non-filaire -, permettent aussi bien de contrôler la dose totale d'exposition (DTE), que de modéliser la lumière, intervenant sur le temps, l'ouverture du flux lumineux, le contour et la température de couleur (Ezrati, 2018). De ce fait, les sources LED et les protocoles dynamiques ouvrent à l'éclairage un champ inédit d'expérience dans le domaine de l'exposition. Cette perspective concerne de nombreux acteurs : concepteurs lumière, scénographes, muséographes, architectes, responsables de la conservation des collections, responsables techniques et informatiques, commissaires d'exposition.

Ces nouvelles technologies permettent-elles aussi une nouvelle forme de médiation? Peut-on créer des ambiances, accompagner et guider le visiteur, délimiter son regard, indiquer ce dont on parle, circonscrire ce que l'on montre et même s'en servir pour rénover les espaces des collections? La modélisation de la lumière peut-elle faire vivre une histoire au visiteur du musée, au même titre qu'un discours, écrit ou oral ? Plus généralement, quels sont les effets possibles de l'éclairage dynamique? En d'autres termes, au-delà "d'éclairer» les œuvres, peut-on aussi penser l'éclairage comme une intention stratégique communicationnelle qui vise à stimuler et à orienter la production de sens des visiteurs? Pour comprendre les enjeux médiatiques de la lumière, nous avons réalisé une étude s'attachant à explorer les effets de l'éclairage dynamique sur l'expérience et la construction de sens des visiteurs ${ }^{1}$. 
Nef du hall, niveau I de la Grande Galerie de l'Évolution. Trois séquences du cycle nycthéméral. À gauche, les tons blancs évoquent l'ambiance lumineuse du jour. Au centre, les tons roses reproduisent l'ambiance du coucher du soleil. À droite, les tons bleus simulent l'ambiance lunaire de la nuit.
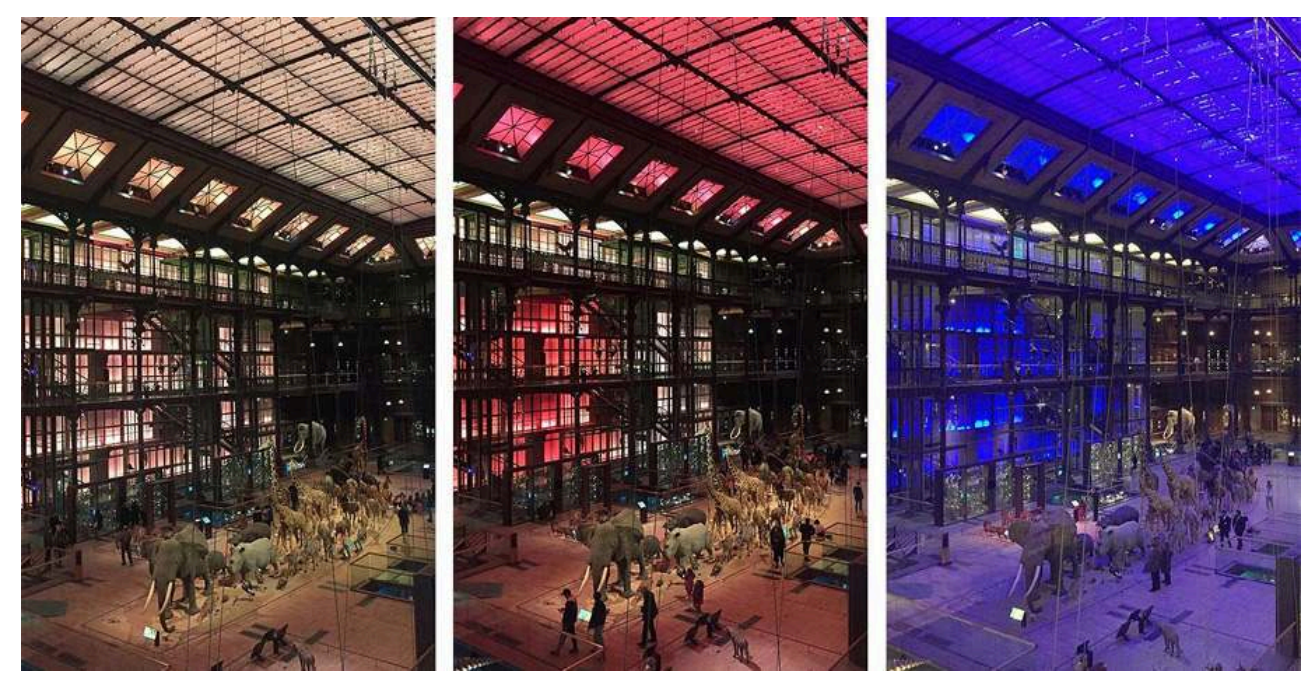

(C) V. Gobbato

\section{En quête du sensible}

La Grande Galerie de l'Évolution à Paris est un terrain propice à notre question de recherche (Gobbato et al., 2020). Elle est l'un des rares musées européens à disposer d'une installation d'éclairage dynamique DMX (Digital Multiplexing). L'installation varie ici au cours du temps, en intensité lumineuse et en température de couleur (voir encadré). Notre étude aborde plusieurs questions de recherche : l'éclairage dynamique de la Grande Galerie de l'Évolution participe-t-il de façon directe ou indirecte à la construction de sens des visiteurs? Et si oui, dans quelle mesure peut-on qualifier les apports de l'éclairage en tant que dispositif de médiation? Comment influence-il l'expérience esthétique, la perception sensorielle, émotionnelle et cognitive de la réception?

Or, comment identifier les effets de la dynamique de l'éclairage sur la construction de sens des visiteurs dans le cours de leur visite ? Il est à remarquer que le simple fait d'évoquer la « lumière » par questionnaire ou par entretien, revient à attirer l'attention du visiteur sur une facette de son expérience qu'il n'aurait peut-être pas remarquée ou conscientisée en l'absence, ou en présence du chercheur (Gobbato, 2019). Il s'agit donc de trouver une méthode de recherche qui permette une visite en situation dite naturelle. Or, on sait bien que l'expérience de visiteurs est difficile à saisir avec les techniques d'enquête habituelles (Davallon \& Flon, 2013). Nous nous sommes ainsi appuyés sur la méthode Remind qui met en œuvre des entretiens en re-situ subjectifs ${ }^{2}$. Ces entretiens permettent d'identifier et d'analyser une partie significative de l'expérience corporelle, cognitive et émotionnelle des visiteurs.

\section{Le spectacle de la Grande Galerie de l'Évolution}

L'installation de la Grande Galerie de l'Évolution a été conçue en 1994 par les architectes Paul Chemetov et Borja Huidobro, le scénographe René Allio et le 
concepteur lumière André Diot. L'intention des concepteurs était de favoriser une " expérience cognitive novatrice, sollicitant des approches multiples à la fois émotionnelles, esthétiques, physiques et sémantiques » (Galangau-Quérat, Chemetov \& Gauthier, 2014). L'installation a été rénovée et enrichie en 2014 par la conceptrice lumière Stéphanie Daniel.

Le projet combine un éclairage muséographique et scénique, et s'apparente à un spectacle son et lumière, reproduisant les couleurs et l'intensité d'une succession de cycles nycthéméraux. Chaque cycle dure environ 75 minutes et simule des effets météorologiques, tels que l'aube, les pluies torrentielles, le soleil du lever au coucher, en passant par le zénith et la nuit (figure. 1). Des projecteurs reproduisent également les couleurs propres à l'environnement des espèces présentées. Ce sont les « univers ", comme les appellent les concepteurs : les pôles, la savane, le désert et la forêt tropicale. Enfin, le projet vise à mettre en valeur la structure architecturale de la Galerie.

Enquête à la Grande Galerie de l'Évolution. À gauche, équipement du visiteur. À droite, entretien en remémoration stimulée.
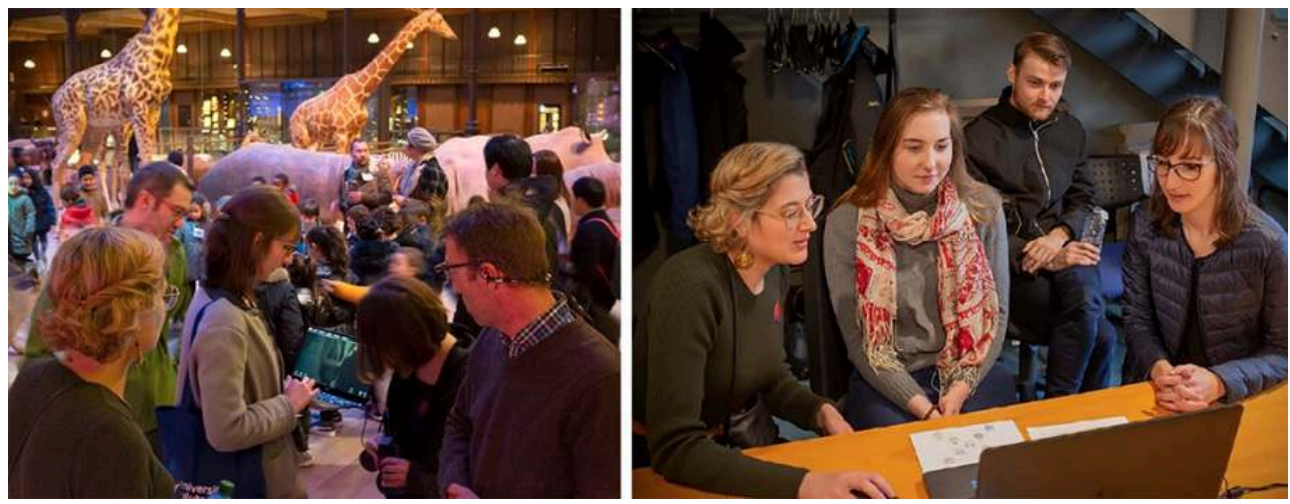

(c) D. Schmitt

\section{La parole au public}

7 Notre enquête a permis de réaliser dix entretiens en remémoration stimulée avec cinq femmes et cinq hommes majeurs dont huit francophones, deux anglophones et sept primo-visiteurs. On cherche à recueillir une description de l'expérience de visite aussi précise que possible. Bien évidemment, les chercheurs s'interdisent de parler de lumière ou de tout autre sujet qui pourrait inciter les visiteurs à parler de l'éclairage. La visite étant libre, elle n'est pas synchronisée avec le cycle nycthéméral. Les visiteurs enquêtés n'ont donc pas nécessairement tous vécu les mêmes séquences, ni assisté aux mêmes phénomènes reproduits par l'éclairage dynamique. Notre étude a néanmoins permis d'identifier quatre séquences significatives de l'expérience des visiteurs en relation avec la lumière. 


\section{S'abstraire du monde extérieur}

8 Une grande majorité des visiteurs évoque avoir perçu au cours de la visite, une ambiance générale caractérisée par des faisceaux de lumière et des zones sombres. Les verbalisations associent cette ambiance au fait de vivre une expérience paisible, de détente, mais aussi aux notions de calme, de sécurité et de confiance. Les visiteurs font aussi référence à un état de recentrement sur soi, de concentration sur les expôts (les spécimens, les œuvres, les dispositifs), et de coupure avec la réalité extérieure. De plus, ils affirment faire appel à leur imaginaire et à leurs souvenirs : l'ambiance lumineuse déclenche des images mnémoniques familières - comme des voyages ou d'autres expériences muséales -, qui concourent à la construction de repères pendant la visite. Il en ressort que le dispositif d'éclairage dynamique de la Grande Galerie stimule les sens par une dimension de bien-être et de contemplation esthétique et méditative. Il favorise une forme d'abstraction du monde externe et de création "d'un lieu à part ", propre au monde de l'exposition. Autrement dit, cette atmosphère immersive, vivante, et riche en variations est suffisamment intense au point de faire oublier à la majorité des visiteurs la réalité extérieure.

Les couleurs du spectacle de la Grande Galerie de l'Évolution ont une influence directe sur la perception esthétique du pelage et des peaux des spécimens.

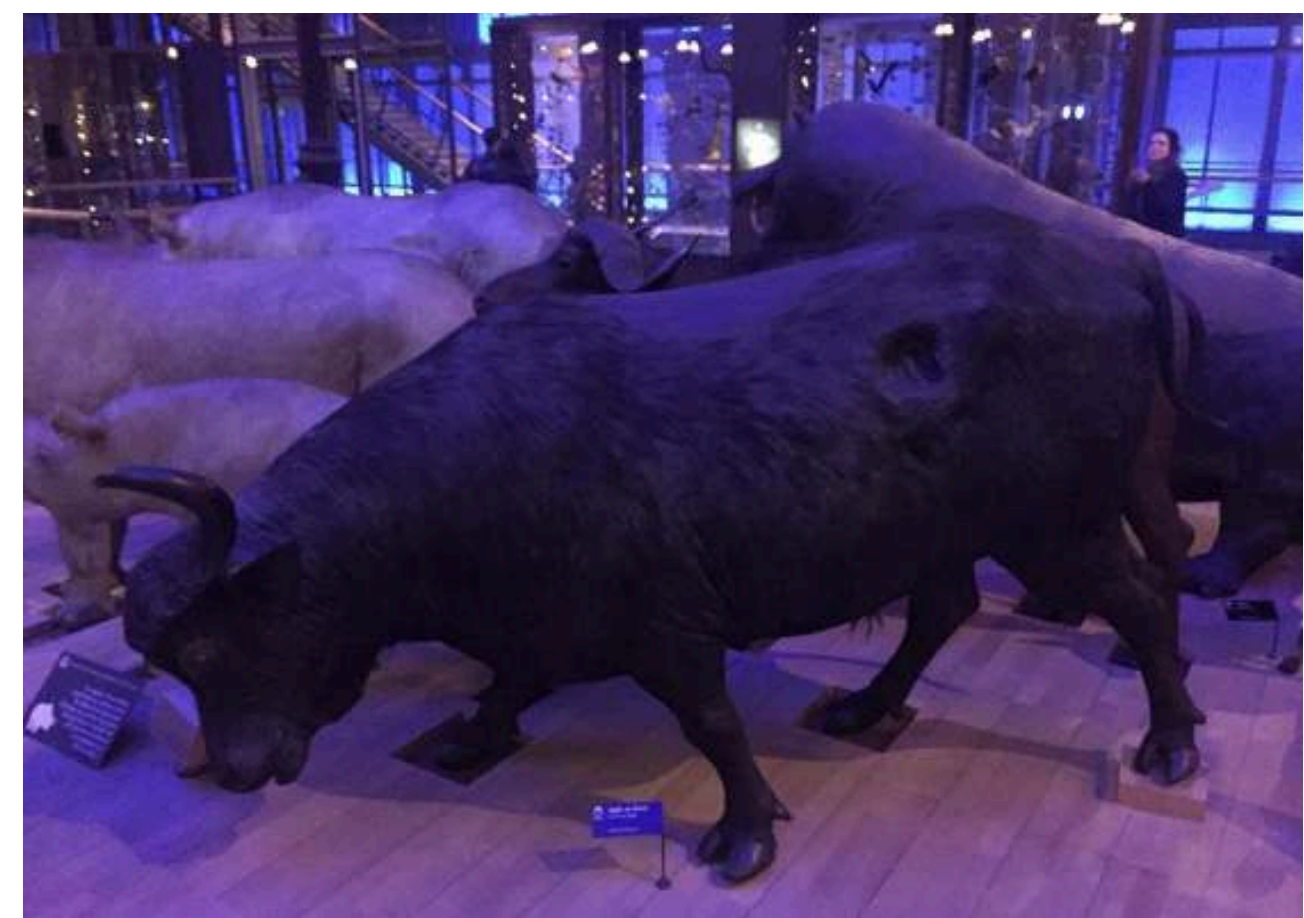

(c) V. Gobbato

\section{Une exposition en technicolor}

9 Les visiteurs évoquent également la mise en scène et les jeux de lumière colorée. La plupart sont sensibles au noir de l'ambiance générale, au jaune, rouge et orange des « univers » dans le hall du niveau I et au bleu de l'étage marin. Ces couleurs participent de la construction du sens à plusieurs degrés. Elles ont un impact sur la perception des 
spécimens comme par exemple s'approcher des animaux et observer leur pelage ou leur peau, ce qui contribue à leur appréciation esthétique. Les couleurs interviennent aussi dans la recontextualisation des spécimens naturalisés, car elles recréent les teintes de leurs environnements de provenance. De plus, en segmentant le parcours de visite et en rythmant l'agencement de l'exposition, les couleurs favorisent l'immersion des visiteurs et la lecture de l'architecture. Elles évoquent d'autres images mnémoniques propres aux expériences passées du visiteur, des voyages, des documentaires, des films, des souvenirs d'enfance, et cela est mobilisé pour comprendre l'exposition. Enfin, les couleurs peuvent induire des sensations corporelles comme le chaud et le froid, en fonction des « univers».

\section{Voir dans l'obscurité : entre fascination et contrainte}

10 L'assombrissement de l'exposition provoque deux réactions opposées chez les visiteurs enquêtés - la fascination et la contrainte -, et cela au cours d'une même expérience de visite. Ce phénomène semble s'expliquer par les différentes nuances de la dimension du sombre. Premièrement, la "pénombre» de l'ambiance générale apaise le regard, fascine et incite à l'imagination. Deuxièmement, le contraste des «ombres" attire l'attention, rythme la découverte et sculpte les spécimens. Enfin, certains espaces laissés dans "l'obscurité » et faiblement éclairés, comme les vitrines et les cartels, peuvent irriter et favoriser un changement d'attention. Les visiteurs quittent rapidement ces endroits et se concentrent sur des espaces visuellement plus ergonomiques. Dans cette perspective, assombrir pour «mieux voir» est vrai, à condition que le niveau d'éclairement soit suffisant pour « voir » les expôts. Ce constat est indépendant de l'âge des visiteurs, alors que l'on aurait tendance à penser que l'assombrissement ne gêne que les personnes âgées.

\section{Intriguer avec la lumière}

11 Nous avons également observé un phénomène en lien avec la manifestation sonore et lumineuse de l'orage, le cycle le plus scénarisé par le dispositif d'éclairage dynamique. Seuls deux visiteurs y ont assisté, néanmoins l'orage a attiré et capté leur l'attention et a fait office de catalyseur d'expérience en cours de visite. D'une part, le dispositif encourage les relations interpersonnelles entre le visiteur et ses accompagnateurs. Le visiteur veut résoudre l'intrigue liée à la réalité de l'orage - est-ce vrai ou pas ? -, et dans cette perspective, il engage une conversation avec son accompagnateur. Cet échange lui permet de disposer d'autres éléments pour construire et stabiliser le sens du phénomène. D'autre part, l'orage incite à la contemplation. Il devient une intrigue, un élément à part entière de l'exposition, et agit sur le ressenti et les sensations. Ces réactions montrent que l'éclairage dynamique peut modifier significativement le cours de la visite en détournant l'attention des expôts, en provoquant un échange entre les publics et en faisant vivre « des sensations fortes ». 
Le spectacle de son et lumière de la Grande Galerie de l'Évolution.

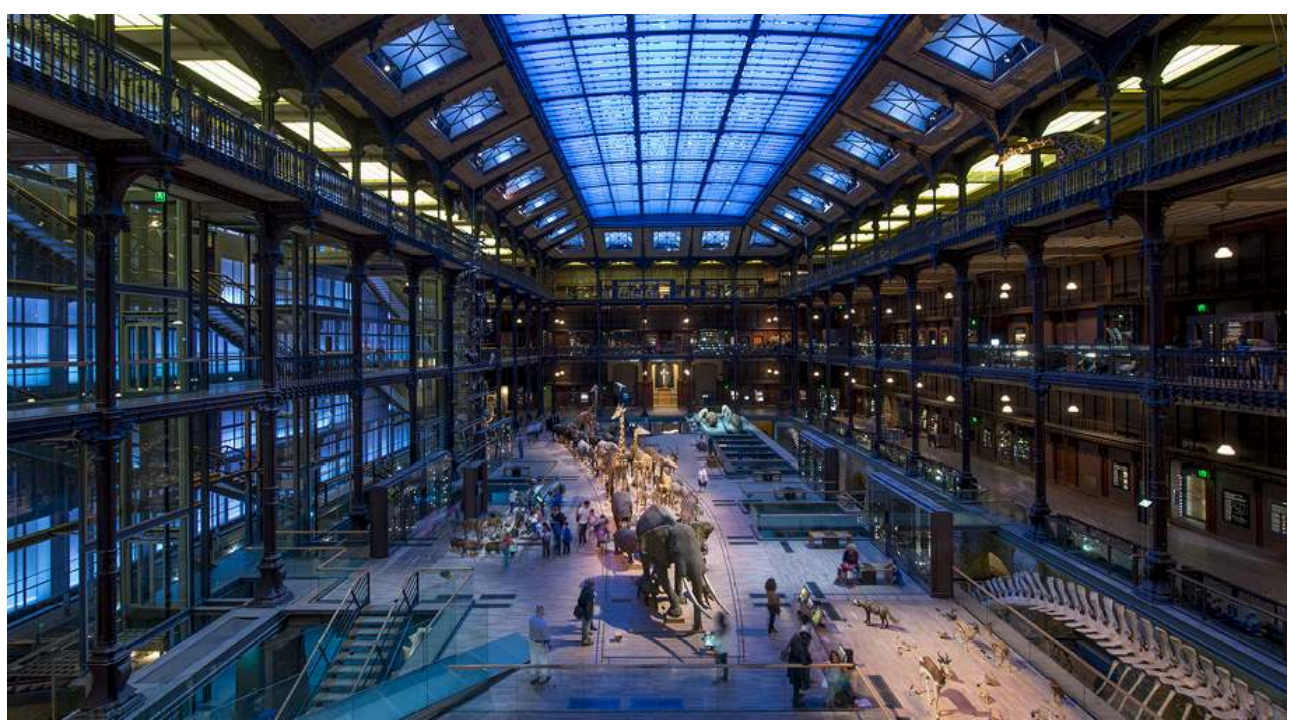

(c) MNHN/B. Jay

\section{Explorer l'étendue des possibles}

12 Les résultats de notre étude exploratoire mettent en évidence l'éclairage dans ses dimensions narratives et médiatiques. Le dispositif lumière est capable de focaliser ou de détourner l'attention, de favoriser des états méditatifs et contemplatifs, de déclencher des sensations et des émotions, d'induire des échanges entre visiteurs, de stimuler des images mnémoniques, de construire l'espace de visite et de participer à la résolution de l'intrigue expographique. L'intention stratégique communicationnelle de cet ensemble scénographique influence les relations entre le public et les objets exposés. Les visiteurs se concentrent sur les spécimens accentués par l'éclairage, et cette proximité leur fait vivre des expériences complémentaires aux dispositifs de médiation déjà en place (écrans, cartels, panneaux graphiques).

En somme, du point de vue de la réception, l'éclairage peut inclure ou exclure, expliciter ou effacer des éléments, aussi bien que favoriser ou empêcher la rencontre avec le contenu et le discours de l'exposition. Cela, au même titre que d'autres dispositifs de médiation écrite (aides à la visite, cartels développés, textes de salles), orale (conférences, visites-guidées, médiations postées), audiovisuelle (audioguide, extraits documentaires, vidéos). L'éclairage favorise aussi un certain degré d'interaction entre les visiteurs, et entre le visiteur et l'espace d'exposition, comme rendu possible par les dispositifs de médiation instrumentée (écrans tactiles, applications, réalité virtuelle et augmentée). Comme le dit Jean-Jacques Ezrati (2014), l'éclairage peut devenir système sémiotique, un co-langage de la grammaire scénographique, une part du discours expographique. Il nous reste à explorer davantage son influence sur l'expérience visiteur et à développer collectivement les formes de médiation de ce co-langage.

Remerciements : les auteurs remercient Sylvie Leleu-Merviel, directrice du laboratoire DeVisu, Virginie Blondeau, Marine Thébault et Marine Lagasse pour leur soutien aux enquêtes, ainsi que 
Éric Joly, Cyril Rouget, Frédérique Lafon, Yves Girault et Elisabeth Vuillemot du Muséum national d'Histoire naturelle pour leur soutien à la réalisation de ce projet.

\section{BIBLIOGRAPHIE}

CIE. Control of damage to museum objects by optical radiation. Technical report, Vienna : CIE 157, 2004, $321 \mathrm{p}$.

Davallon J. \& Flon E. Le média exposition, in Gottesdiener H. \& Davallon J., Culture \& Musées, La muséologie : 20 ans de recherches (numéro hors-série), 2013, pp. 19-37.

Ezrati J.-J. L'éclairage dynamique sous DALI, La lettre de l'Ocim, n¹75, 2018, pp. 22-26.

Ezrati J.-J. Éclairage d'exposition. Musées et autres espaces. Paris : Eyrolles, 2014, 166 p.

Galangau-Quérat F., Chemetov P. \& Gauthier C.-A. La Grande Galerie de l'Évolution. L'esprit du lieu. Paris : Nouvelles Éditions Scala, 2014, 63 p.

Gobbato V., Blondeau V., Thébault M. \& Schmitt D. L'éclairage dynamique, un dispositif de médiation. Le cours d'action pour évaluer l'influence de la lumière sur l'expérience visiteur au musée, Activités, vol. 17 n², 2020 : http://journals.openedition.org/activites/5598

Gobbato V. L'éclairage dynamique, un dispositif de médiation? Mémoire de Muséologie, sous la direction de François Mairesse, Paris : Université Sorbonne Nouvelle, 2019, 336 p.

Schmitt D. \& Meyer-Chemenska M. Expériences de visite : de la transmission à la liction, La lettre de l'Ocim, nº155, 2014, pp. 17-23.

\section{NOTES}

1. L'étude a été réalisée au Muséum national d'Histoire naturelle, Paris, dans la Grande Galerie de l'Évolution, au mois de janvier 2019. Elle a été conçue dans le cadre du mémoire de Master de Viviana Gobbato, en Muséologie et Nouveaux Médias, Université Sorbonne Nouvelle-Paris 3, et a été rendue possible avec le soutien du Laboratoire DeVisu, Université Polytechnique Hauts-deFrance.

2. Voir l'article de Daniel Schmitt et Muriel Meyer-Chemenska dans La lettre de l'Ocim $\mathrm{n}^{\circ} 155$. Pour en savoir plus sur la méthode Remind, une vidéo est disponible sur HAL : https://hal.archivesouvertes.fr/medihal-01899947 


\section{RÉSUMÉS}

La lumière est incontestablement un élément essentiel de la visite muséale. Et depuis une vingtaine d'années, les sources LED et les protocoles dynamiques lui ont ouvert un champ inédit d'expérience. Les auteurs reviennent sur une étude menée auprès des visiteurs de la Grande Galerie de l'Évolution pour appréhender les enjeux médiatiques de la lumière.

\section{INDEX}

Mots-clés : éclairage, muséum d'histoire naturelle

\section{AUTEURS}

\section{VIVIANA GOBBATO}

Doctorante, Université Sorbonne Nouvelle-Paris 3, Laboratoire Cerlis, sous la direction de François Mairesse. Son sujet de recherche porte sur l'éclairage dans les musées. viviana.gobbato@sorbonne-nouvelle.fr

\section{DANIEL SCHMITT}

Maître de conférences HDR, Université Polytechnique Hauts-de-France, Laboratoire DeVisu. Ses recherches portent sur le design d'expérience et les médiations instrumentées dans les musées. daniel.schmitt@uphf.fr 